\title{
A case of laparoscopic partial hepatic S7 resection for postoperative liver metastasis of rectal malignant melanoma
}

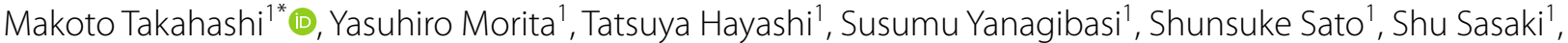 \\ Kunio Takuma ${ }^{1}$ and Haruka Okada²
}

\begin{abstract}
Background: Anorectal malignant melanoma (ARMM) has an extremely poor prognosis, and there is no report of resection of liver metastases so far. We report herein a rare case of postoperative laparoscopic partial hepatic S7 resection for rectal malignant melanoma.

Case presentation: A 51-year-old female patient with a diagnosis of an ARMM underwent a laparoscopic rectal amputation. Eleven months later, computed tomography (CT) revealed a 14-mm nodule in liver segment 7 (S7), which was diagnosed as a hepatic recurrence of the ARMM. Because no other recurrences were found, a laparoscopic partial resection of S7 was performed. Pathological analysis found intracellular melanin deposition, and immunostaining was S-100 (+), HMB-45 (+), and SOX-10 (+). Based on these findings, a liver metastasis of malignant melanoma was diagnosed. The patient is alive 7 months after the second surgery and has so far experienced no recurrences.
\end{abstract}

Conclusion: We reported an extremely rare case of a laparoscopic resection of a liver metastasis following surgery for ARMM.

Keywords: Anorectal malignant melanoma, Liver metastasis, Laparoscopic surgery

\section{Background}

Anorectal malignant melanoma (ARMM) is a rare disease, accounting for about $1 \%$ of all malignant melanoma cases and $0.5-2 \%$ of all anorectal malignancies [1]. It is highly malignant, with a 5 -year survival rate of $25 \%$ and a median survival of 16 to 28 months [2-4]. Even if radical resection is performed for ARMM, recurrences in the liver and lung are highly likely. Herein, we described a rare case of a laparoscopic resection of a liver metastasis following surgery for ARMM.

\footnotetext{
*Correspondence: makoto_6_c@yahoo.co.jp

1 Department of Surgery, Tokyo Metropolitan Tama Medical Center, 2-8-29 Musashidai, Fuchu, Tokyo 183-8524, Japan

Full list of author information is available at the end of the article
}

\section{Case presentation}

A 51-year-old female patient with a history of bipolar disorder and hyperlipidemia underwent a colonoscopy after a fecal occult blood test returned positive. Colonoscopy revealed a $30-\mathrm{mm}$-sized semi-pedunculated tumor in the lower rectum $(\mathrm{Rb})$ and a black protruding lesion extending upwards into the anal canal. Based on these findings, ARMM was diagnosed (Fig. 1a, b). A laparoscopic rectal amputation was performed, and pathological analysis of tissue specimens revealed peripheral discontinuous melanin deposition (Fig. 2a, b). The tumor had invaded the submucosa, and one lymph node metastasis was detected. Histopathological findings indicated diffuse proliferation of atypical cells with prominent nuclei of varying size accompanied by melanin deposition (Fig. 2c). Immunostaining was positive for HMB-45 (Fig. 2d), S-100 (Fig. 2e), and SOX-10 (Fig. 2f). Based on 


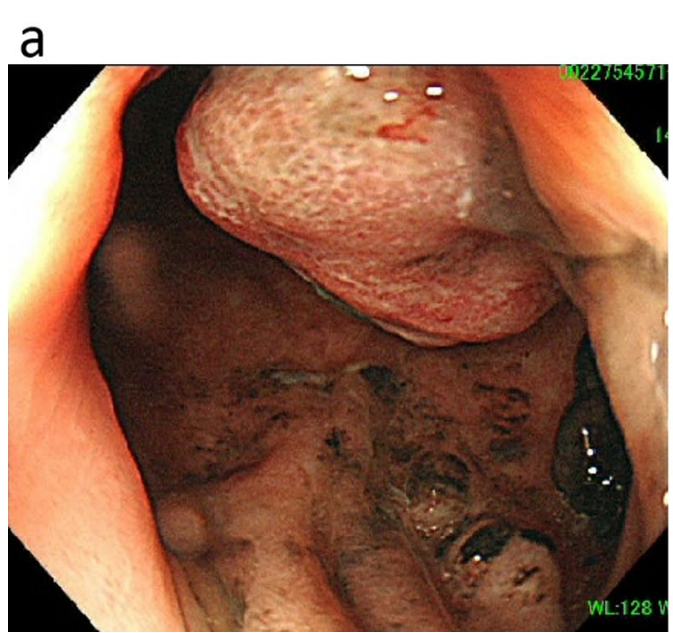

\section{b}

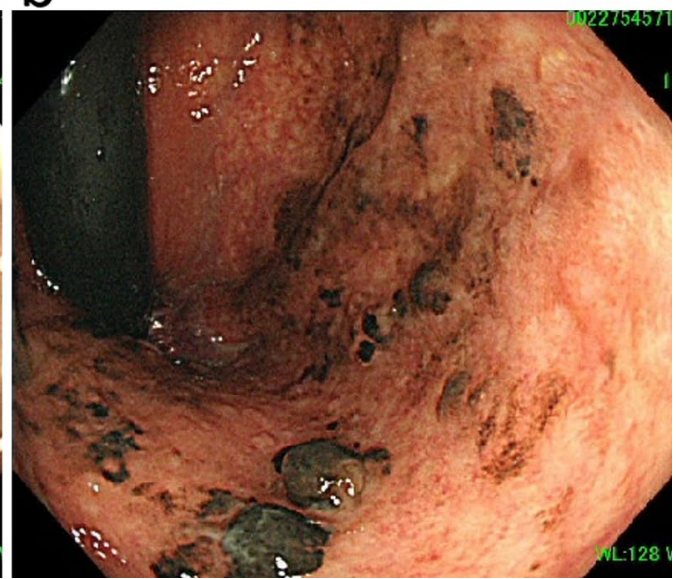

Fig. 1 Colonoscopy. a Sub-pedunculated tumor found in the lower rectum (Rb). b Black protruding lesion found extending upwards into the anal canal
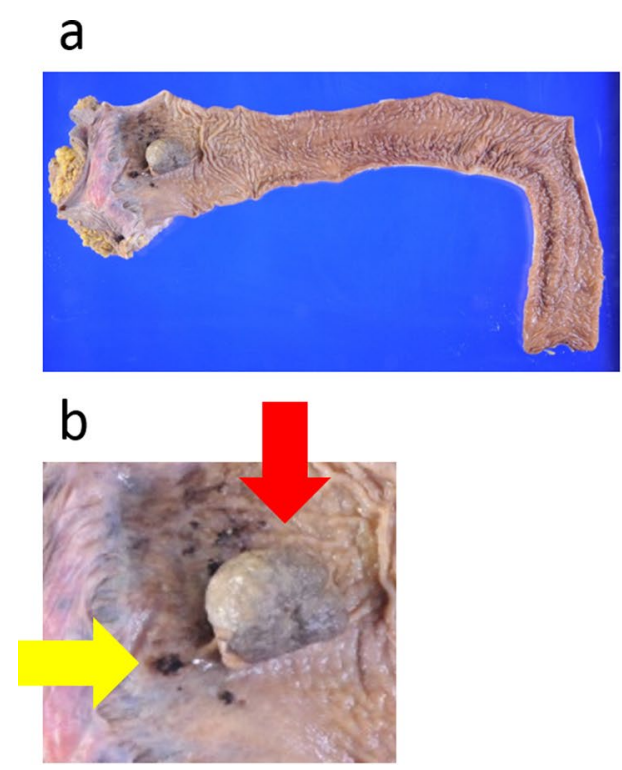

\section{C}
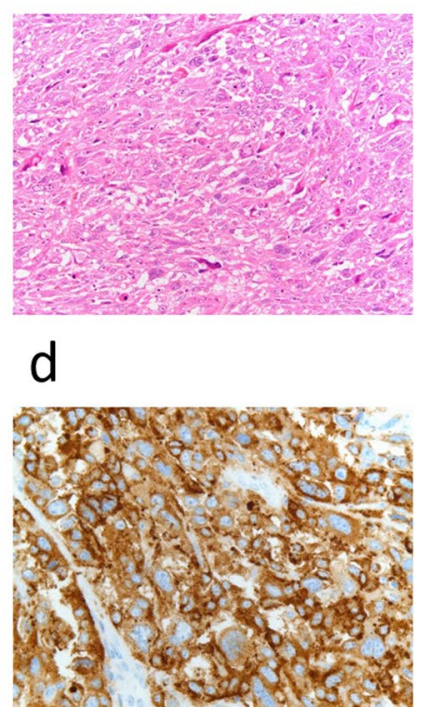

$\mathrm{e}$

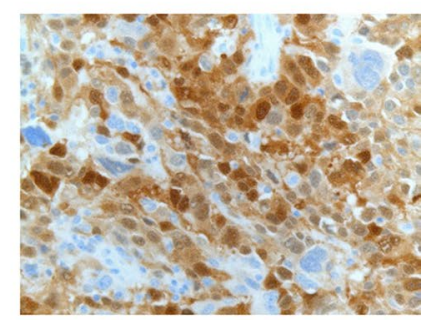

f

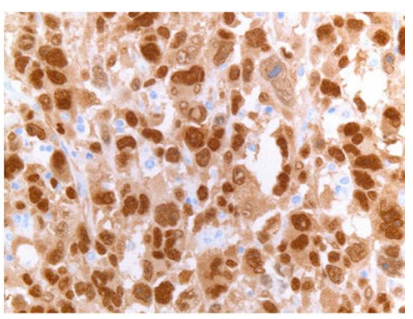

Fig. 2 Macroscopic findings, histopathological findings, and immunostaining findings of ARMM. a, b Pathological specimens revealed a semi-pedunculated tumor $30 \mathrm{~mm}$ in size in the anal canal and peripheral discontinuous melanin depositions. c Histopathological findings demonstrated diffuse proliferation of atypical cells with prominent nuclei of varying size accompanied by melanin depositions. $\mathbf{d}$ Positive for HMB-45. e Positive for S-100. f Positive for SOX-10

these findings, stage III ARMM was diagnosed, and the patient was followed up without postoperative adjuvant chemotherapy.

At postoperative months 3 and 8 , CT revealed no recurrence. At postoperative month 11, CT revealed a 14-mm-diameter tumor with a faint contrast effect at the margin of S7 (Fig. 3a). The tumor showed low signal intensity in the hepatocellular phase of gadolinium-ethoxybenzyl-diethylenetriaminepentaacetic acid
(Gd-EOB-DTPA)-enhanced magnetic resonance imaging (MRI) (Fig. 3b), and positron emission tomography (PET)-CT indicated a large accumulation of FDG (Fig. 3c). Based on these findings, a liver metastasis of ARMM was diagnosed.

Surgical resection is recommended for postoperative oligometastatic recurrences of malignant melanoma [5]. In the present patient, a single liver metastasis recurred 11 months after surgery for the primary lesion. 
a

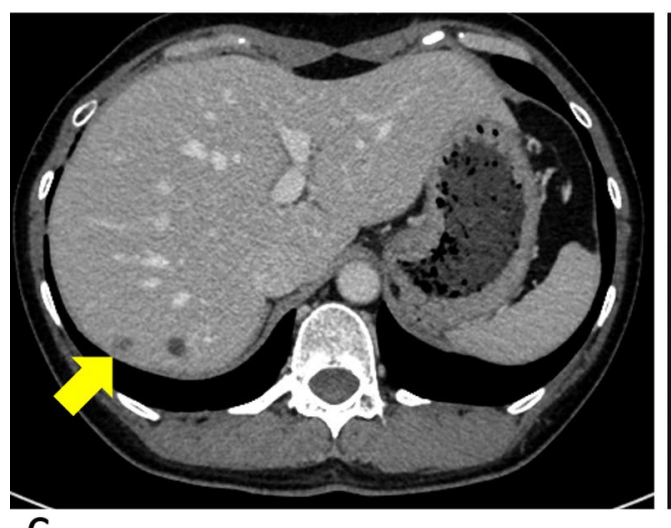

b

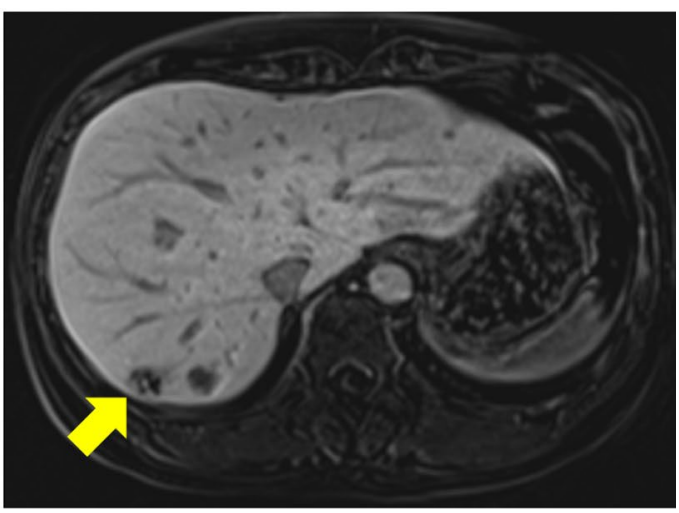

C

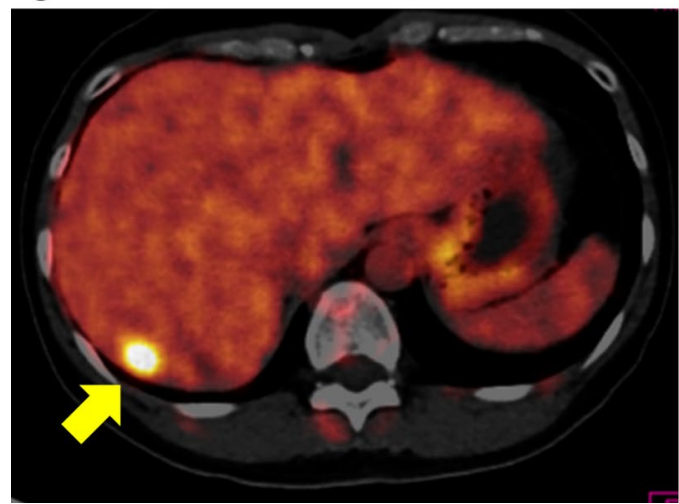

Fig. 3 Imaging findings of the liver metastasis. a CT demonstrated a 14-mm-sized tumor with a faint contrast effect on the margin of liver S7. b The tumor showed a low signal intensity in the hepatocellular phase of Gd-EOB-DTPA-enhanced MRI. c The tumor showed strong accumulation of FDG on PET-CT

A complete resection was possible via minimally invasive laparoscopic surgery; therefore, we decided to perform a laparoscopic partial resection of S7. The operative time was $4 \mathrm{~h} 8 \mathrm{~min}$, and the amount of bleeding was $30 \mathrm{~g}$. The postoperative course was uneventful, and the patient was discharged on postoperative day 7 .

Macroscopically, a 16-mm solid nodule was observed together with melanin deposition (Fig. 4a-c). Immunostaining was positive for HMB-45 (Fig. 4d), S-100 (Fig. 4e), and SOX-10 (Fig. 4f), confirming the diagnosis of a liver metastasis of ARMM. Nivolumab was started as adjuvant chemotherapy, and 7 months after the surgery for the metastasis, the patient is still alive without any recurrences.

\section{Discussion}

Malignant melanoma is a mesenchymal tumor originating in melanocytes. It may occur in the skin, retina, head and neck area or gastrointestinal tract [4]. ARMM is a rare disease, accounting for $1 \%$ of malignant melanoma cases and $0.5-2 \%$ of anorectal malignancies [1].
The prognosis of ARMM is poor, with a 5-year survival rate of $25 \%$ and a median survival of $16-28$ months [1, 2].

For malignant melanoma with distant metastases, the first-line treatment is chemotherapy, including targeted drugs and immune checkpoint inhibitors. When nivolumab, an anti-PD-1 antibody, was administered to patients with malignant melanoma with distant metastases, the 1-year survival rate was $72.9 \%$ [6]. When ipilimumab, an anticytotoxic T-lymphocyte antigen-4 (anti-CTLA-4) monoclonal antibody, was administered at $10 \mathrm{mg} / \mathrm{kg}$ to a similar group of patients, the median overall survival was 15.7 months and the 5-year survival rate was $25 \%$ [7].

On the other hand, surgical resection may be considered for a distant oligometastatic recurrence of malignant melanoma if total resection of the lesion is possible [5, 8-11]. In such cases, the 1-year survival rate is reportedly $51 \%$, and the 2 -year survival rate is $38 \%$ [5]. Patients in past reports who underwent a complete resection of liver metastases of malignant 
a

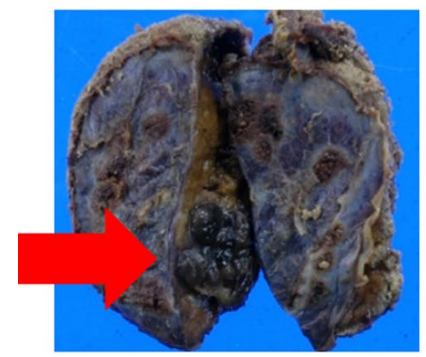

b

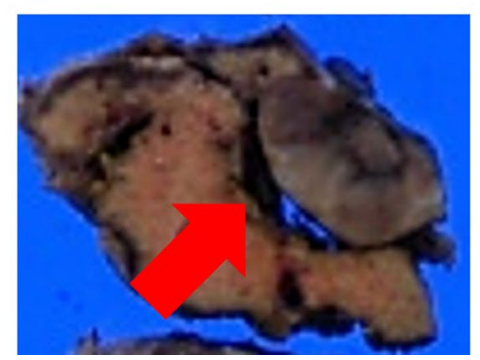

C
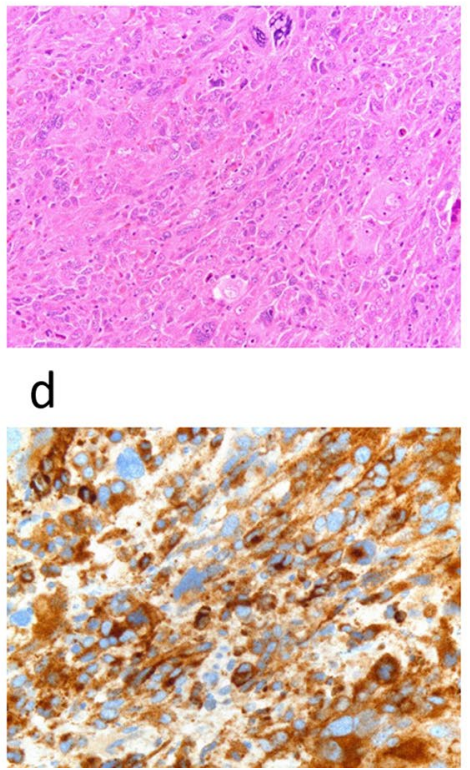

e

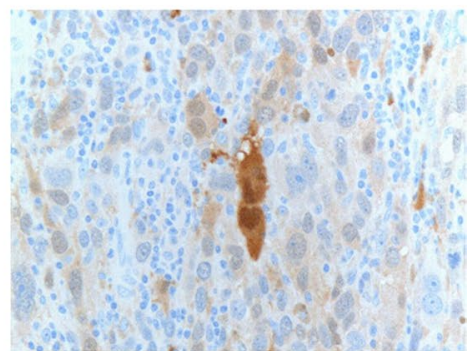

$f$

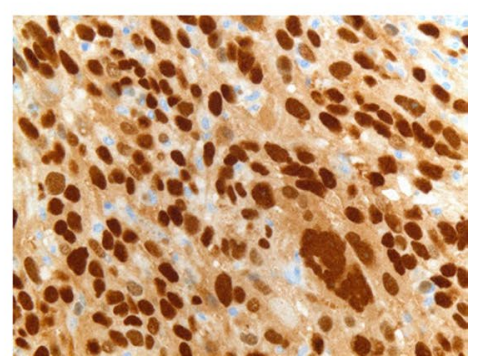

Fig. 4 Macroscopic findings, histopathological findings, and immunostaining findings of the liver metastasis. a, b Pathological specimens revealed a 16-mm-diameter solid nodule accompanied by melanin depositions. c Histopathological findings demonstrated diffuse proliferation of atypical cells with prominent nuclei of varying size accompanied by melanin deposition. d Positive for HMB-45. e Positive for S-100. f Positive for SOX-10

melanoma had a median survival time of 27.7 months and a 5-year survival rate of $33 \%$ [8].

Although some studies have reported resection of liver metastases of malignant melanoma [12, 13], none so far have reported resection of a liver metastasis of ARMM.

In the present instance, surgery was chosen as the treatment modality because the lesion was a single oligometastatic recurrence of liver metastasis occurring 11 months after resection of the primary ARMM lesion. The procedure was able to be performed via laparoscopic surgery, which is less invasive than other surgical techniques.

Nivolumab has been shown to be effective as postoperative adjuvant therapy in patients with melanoma with completely resected lymph node metastases or distant metastases. In a previous study where nivolumab was given as adjuvant chemotherapy for one year after complete resection of stage III and IV malignant melanoma, the recurrence-free survival rate was $51.7 \%$ and the overall survival rate was $77.9 \%$ after 4 years [14].

In recent years, various anticancer agents and molecular-targeted agents have appeared in the adjuvant chemotherapy arsenal against malignant melanoma, including dabrafenib plus trametinib $[15,16]$ and pembrolizumab [17]. In the treatment of ARMM, multidisciplinary treatment combining surgery and chemotherapy for metastatic lesions has the potential to improve the prognosis.

\section{Conclusions}

We reported a case of ARMM in which the primary lesion was resected laparoscopically. A liver metastasis was also subsequently resected laparoscopically. This is the first report of resection of liver metastases of ARMM. Although the prognosis of ARMM is poor, surgery should be considered in cases of oligometastasis.

\section{Acknowledgements \\ Not applicable.}

\section{Authors' contributions}

MT drafted the manuscript. KT, SY and SS performed the first operation. MT, YM, and SS performed the second operation. TH and SS contributed to the preoperative examination and diagnosis. KT followed up the patient. $\mathrm{HO}$ diagnosed the disease pathologically. All the authors read and approved the final manuscript.

Funding

None.

Availability of data and materials

Data sharing is applicable to this article.

\section{Declarations}

Ethics approval and consent to participate Not applicable.

\section{Consent for publication}

Informed consent was obtained from the patient for the publication of this case report. 


\section{Competing interests}

The authors declare that they have no competing interests.

\section{Author details}

${ }^{1}$ Department of Surgery, Tokyo Metropolitan Tama Medical Center, 2-8-29 Musashidai, Fuchu, Tokyo 183-8524, Japan. ${ }^{2}$ Department of Pathology, Tokyo Metropolitan Tama Medical Center, Tokyo, Japan.

Received: 3 April 2021 Accepted: 19 October 2021

Published online: 26 October 2021

\section{References}

1. Khan M, Bucher N, Elhassan A, Barbaryan A, Ali AM, Hussain N, et al. Primary anorectal melanoma. Case Rep Oncol. 2014;7(1):164-70.

2. Latteri S, Teodoro M, Malaguarnera M, Mannino M, Currò G, La Greca G. Abdominal perineal resection or wilde local excision in primary anorectal malignant melanoma. Case report and review. Ann Med Surg (Lond). 2017;19:74-7.

3. Callahan A, Anderson WF, Patel S, Barnholtz-Sloan JS, Bordeaux JS, Tucker MA, et al. Epidemiology of anorectal melanoma in the United States: 1992 to 2011. Dermatol Surg. 2016;42(1):94-9.

4. Mastoraki A, Schizas D, Ntella V, Roka A, Vailas M, Papanikolaou IS, et al. Clinical evidence, diagnostic approach and challenging therapeutic modalities for malignant melanoma of the anorectum. ANZ J Surg. 2020. https://doi.org/10.1111/ans.16497.

5. Deutsch GB, Flaherty DC, Kirchoff DD, Bailey M, Vitug S, Foshag LJ, et al. Association of surgical treatment, systemic therapy, and survival in patients with abdominal visceral melanoma metastases, 1965-2014: relevance of surgical cure in the era of modern systemic therapy. JAMA Surg. 2017;152(7):672-8.

6. Robert C, Long GV, Brady B, Dutriaux C, Maio M, Mortier L, et al. Nivolumab in previously untreated melanoma without BRAF mutation. N Engl J Med. 2015:372(4):320-30

7. Ascierto PA, Del Vecchio M, Mackiewicz A, Robert C, Chiarion-Sileni $\checkmark$, Arance A, et al. Overall survival at 5 years of follow-up in a phase III trial comparing ipilimumab 10 mg/kg with $3 \mathrm{mg} / \mathrm{kg}$ in patients with advanced melanoma. J Immunother Cancer. 2020;8(1)
8. Wood TF, DiFronzo LA, Rose DM, Haigh PI, Stern SL, Wanek L, et al. Does complete resection of melanoma metastatic to solid intra-abdominal organs improve survival? Ann Surg Oncol. 2001;8(8):658-62.

9. Harpole DH Jr, Johnson CM, Wolfe WG, George SL, Seigler HF. Analysis of 945 cases of pulmonary metastatic melanoma. J Thorac Cardiovasc Surg. 1992;103(4):743-8.

10. Tafra L, Dale PS, Wanek LA, Ramming KP, Morton DL. Resection and adjuvant immunotherapy for melanoma metastatic to the lung and thorax. J Thorac Cardiovasc Surg. 1995;110(1):119-28.

11. Schuhan C, Muley T, Dienemann H, Pfannschmidt J. Survival after pulmonary metastasectomy in patients with malignant melanoma. Thorac Cardiovasc Surg. 2011;59(3):158-62.

12. Muraki R, Morita Y, Ida S, Kitajima R, Furuhashi S, Kiuchi R, et al. Multimodal therapy with surgery and adjuvant nivolumab for late-onset multiple liver metastases of choroidal malignant melanoma: a case report. Surg Case Rep. 2020;6(1):187.

13. Aubin JM, Rekman J, Vandenbroucke-Menu F, Lapointe R, Fairfull-Smith RJ, Mimeault R, et al. Systematic review and meta-analysis of liver resection for metastatic melanoma. Br J Surg. 2013;100(9):1138-47.

14. Ascierto PA, Del Vecchio M, Mandalá M, Gogas H, Arance AM, Dalle S, et al. Adjuvant nivolumab versus ipilimumab in resected stage IIIB-C and stage IV melanoma (CheckMate 238): 4-year results from a multicentre, double-blind, randomised, controlled, phase 3 trial. Lancet Oncol. 2020;21(11):1465-77.

15. Long GV, Hauschild A, Santinami M, Atkinson V, Mandalà M, ChiarionSileni $V$, et al. Adjuvant dabrafenib plus trametinib in stage III BRAFmutated melanoma. N Engl J Med. 2017;377(19):1813-23.

16. Hauschild A, Dummer R, Schadendorf D, Santinami M, Atkinson V, Mandalà $\mathrm{M}$, et al. Longer follow-up confirms relapse-free survival benefit with adjuvant dabrafenib plus trametinib in patients with resected BRAF V600-mutant stage III melanoma. J Clin Oncol. 2018:36(35):3441-9.

17. Eggermont AMM, Blank CU, Mandala M, Long GV, Atkinson V, Dalle S, et al. Adjuvant pembrolizumab versus placebo in resected stage III melanoma. N Engl J Med. 2018;378(19):1789-801.

\section{Publisher's Note}

Springer Nature remains neutral with regard to jurisdictional claims in published maps and institutional affiliations.

\section{Submit your manuscript to a SpringerOpen ${ }^{\circ}$ journal and benefit from:}

- Convenient online submission

- Rigorous peer review

- Open access: articles freely available online

- High visibility within the field

- Retaining the copyright to your article

Submit your next manuscript at $\boldsymbol{\nabla}$ springeropen.com 